\title{
Infección por Trypanosoma Cruzi en donantes de un banco de sangre del departamento Boyacá, Colombia
}

\author{
Sandra Helena Suescún Carrero ${ }^{\text {a.1.2; }}$ Mabel Idaliana Medina Alfonso ${ }^{\text {b.1.2. }}$;ubia Alexandra Murcia García ${ }^{\text {c; }}$
} Sandra Marina Forero Pulido ${ }^{d}$

a Bacterióloga. Estudiante de Doctorado en Metodología de la Investigación y Salud Pública. ORCID: https://orcid.org/0000-0001-5821-4421, sandrahsc@ yahoo. com; ${ }^{b}$ Bacterióloga. Magíster en Sistemas Integrados de Gestión de Calidad. ORCID: https://orcid.org/0000-0002-9264-6516, mabel.medina@boyaca.gov.com ; ${ }^{\mathrm{c}}$ Bacterióloga. Especialista en Gerencia de Instituciones en Salud, Hemocentro del Centro Oriente Colombiano- HCOC, Tunja, Colombia. ORCID: https://orcid.

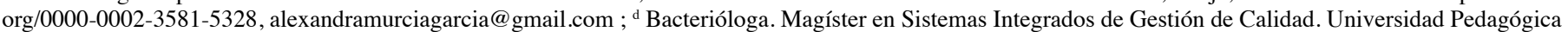
y Tecnológica de Colombia. Tunja, Colombia. ORCID: https://orcid.org/0000-0003-2118-1157, safopu@gmail.com;

1 Investigación del Laboratorio Departamental de Salud Pública’s Research Group. 2 Secretaría de Salud de Boyacá Tunja, Colombia. DOI $10.22517 / 25395203.24601$

\section{Resumen}

Introducción: La Enfermedad de Chagas también conocida como Tripanosomiasis americana es un problema de salud pública. Se calcula que en el mundo hay entre 6 y 7 millones de personas infectadas por Trypanosoma cruzi, la mayoría de ellas en América Latina.

Objetivo: Identificar la prevalencia de infección por Trypanosoma cruzi en donantes de un banco de sangre del departamento de Boyacá 2016-2018.

Materiales y métodos: Estudio descriptivo retrospectivo realizado en 25.920 donantes de sangre del departamento de Boyacá. La fuente de información fue secundaria. Se determinó la prevalencia de la infección por Trypanosoma cruzi y se compararon según sexo, grupo etario y tipo de donante a través de análisis de frecuencias.

Resultados: Se evaluaron 9187 donantes durante el año 2016; 8517 en el 2017 y 8216 en el 2018, de los cuales $56,1 \%$ eran de sexo femenino y $43,9 \%$ masculino. La prevalencia de la infección por Trypanosoma cruzi fue $0,17 \%$ en la tamización y $0,08 \%$ con las pruebas confirmatorias de las cuales el 70,0\% eran mujeres, el $85,0 \%$ donantes voluntarios por primera vez, el rango de edad en el que se presentó mayor prevalencia de este marcador serológico fue de 41 a 50 y de 51 a 65 años con un $35,0 \%$ cada uno . La prevalencia de la infección de Chagas presentó asociación estadísticamente significativa con la edad $(p<0,05)$.

Conclusiones: Los bancos de sangre resultan ser una de las fuentes de información disponibles para monitorear el comportamiento de la enfermedad de Chagas y evaluar la toma de decisiones en salud pública, teniendo en cuenta que la enfermedad se caracteriza de manera asintomática y sin compromiso clínico en la mayoría de los casos.
Palabras clave: Bancos de sangre, Trypanosoma cruzi, Transfusión sanguínea, Prevalencia, Marcadores séricos.

\section{Prevalence of Trypanosoma cruzi infection in donors from a blood bank in Boyacá, Colombia}

\begin{abstract}
Introduction: Chagas Disease, also known as American Trypanosomiasis, is a public health problem. It is estimated that in the world there are between 6 and 7 million people infected with Trypanosoma cruzi, most of them in Latin America.
\end{abstract}

Objectives: To identify the prevalence of Trypanosoma cruzi infection in donors from a blood bank in the department of Boyacá 2016-2018.

Materials and methods: Retrospective-descriptive study carried out in 25,920 blood donors from the department of Boyacá (Colombia). The source of information was secondary. The prevalence of Trypanosoma cruzi infection was determined and compared according to sex, age group and type of donor through frequency analysis.

Results: 9187 donors were evaluated during 2016; 8,517 in 2017 and 8,216 in 2018, of which $56.1 \%$ were female and $43.9 \%$ male. The prevalence of Trypanosoma cruzi infection was $0.17 \%$ in screening and $0.08 \%$ with confirmatory tests of which $70.0 \%$ were women, $85.0 \%$ first-time volunteer donors, the range of The age at which the highest prevalence of this serological marker was found was 41 to 50 and 51 to 65 years with $35.0 \%$ each. The prevalence of Chagas infection showed a statistically significant association with age $(\mathrm{p}<0.05)$.

Conclusions: Blood banks turn out to be one of the sources of information available to monitor the behavior 
of Chagas disease and evaluate decision-making in public health, taking into account the characteristics of the disease in which the majority of cases they are asymptomatic and without clinical compromise.

Keywords: Blood banks; Trypanosoma cruzi; Blood Transfusion; Prevalence; Serum markers.

\section{Introducción}

La Enfermedad de Chagas (EC), también conocida como Tripanosomiasis americana, es una enfermedad parasitaria causada por el flagelado Trypanosoma cruzi ( $T$ cruzi), de la familia Trypanosomatidae, el agente etiológico fue identificado por primera vez en Brasil.

La EC está en la lista de enfermedades desatendidas por la Organización Mundial de la Salud (OMS) si bien representa un problema de salud pública en los países de centro y sur América. La EC se relaciona estrechamente con problemáticas sociales como la pobreza, malas condiciones de salubridad y falta de acceso al sistema de salud (1).

En los últimos años, los estudios de transmisión del parásito incluyen otras vías diferentes a la producida por la picadura del vector. Se ha descrito el riesgo que existe en procedimientos como transfusión de sangre y trasplantes de órganos, se consideran como la segunda fuente de transmisión de $T$ cruzi. Otras formas descritas son la trasmisión de madre a hijo durante el período gestacional y, como caso aislado, por accidente laboral (2).

A medida que se progresa en el control vectorial, otras fuentes de infección, como la transmisión por transfusiones, cobran mayor relevancia, sobre todo si se considera que en las zonas urbanas del continente habita más del 70\% de la población y la mayor parte está compuesta por inmigrantes que han pasado sus primeros años de vida en zonas endémicas (3).

La enfermedad de Chagas es una enfermedad emergente en los Estados Unidos de América (EUA), en Canadá y en los países europeos (España, Holanda, Suiza), así como en Australia y Japón $(4,5)$. Los movimientos migratorios en busca de empleo de los habitantes de zonas rurales donde la infección es más frecuente, los llevan, incluso a las áreas urbanas del mismo país o más allá de las fronteras de Latinoamérica, lo cual ha cambiado considerablemente la epidemiología de la enfermedad, que ha dejado de ser exclusiva de esta región (6)

Se calcula que en el mundo hay entre 6 y 7 millones de personas infectadas por Trypanosoma cruzi, la mayoría de ellas en América Latina (7). La enfermedad de Chagas se encuentra sobre todo en zonas endémicas de 21 países de América Latina. Colombia tiene las cifras más alarmantes en población general de la región Andina después de Bolivia, aunque es importante tener en cuenta que la población de Colombia es 4.6 veces la población de Bolivia.

Se estima que en Colombia el número de infectados es de 437.960, los nuevos casos por año por transmisión vectorial oscilan en 5.274 (1). Durante el año 2016 en Casanare, Santander, Boyacá, Tolima y Arauca se registraron el $79 \%$ de los casos de Chagas en fase crónica (8), en el año 2017 los departamentos que más casos confirmaron fueron Casanare con $24.6 \%$, Bogotá D.C $20 \%$, Arauca $19.5 \%$ y Santander $13.2 \%$, entre estos suman el $77.3 \%$ de los casos confirmados en el país (9).

El objetivo de este trabajo consistió en identificar la prevalencia de infección por Trypanosoma cruzi en donantes de un banco de sangre del departamento de Boyacá 2016-2018 ya que porcentaje de reactividad en donantes para el marcador serológico anti - T.cruzi a nivel nacional para el año 2016 correspondió a $0,27 \%$; $0,19 \%$ en el 2017 y en el $20180,14 \%(10,11,12)$.

\section{Materiales y métodos}

Estudio descriptivo retrospectivo, en el que se estableció la seroprevalencia de la infección de T.cruzi en 25.920 donantes de sangre captados en el departamento de Boyacá por el banco de sangre Hemocentro del Centro Oriente Colombiano (HCOC), durante los años 2016 a 2018 a través del sistema de información de hemovigilancia.

Los datos fueron digitados y depurados en el programa Microsoft Excel ${ }^{\circledR}$, en donde se incluyeron las variables de edad, sexo, tipo de donantes, procedencia, prueba de tamización y pruebas complementarias para $T$ cruzi. La información se recuperó de una fuente secundaria del formato REG-R01.002.5080-017 hemovigilancia informe estadístico mensual de bancos de sangre hasta el año 2017 y del Sistema Información Hemovigilancia SIHEVI-INS ${ }^{\circledR}$ del año 2018.

Para el tamizaje de la infección por Trypanosoma cruzi, el banco de sangre utilizó la técnica quimioluminiscencia; para las pruebas complementarias se empleo la técnica de Inmufluorescencia Indirecta IFI y ELISA Chagas III Ags Totales, siguiendo los lineamientos establecidos del Anexo técnico $\mathrm{N}^{\circ} 2$ y $\mathrm{N}^{\circ} 3$ de la Circular 0082 de 2011 emitida por el Instituto Nacional de Salud y el Ministerio de Salud y Protección Social (13). 


\section{Análisis de variables}

Se realizó un análisis univariado calculando las frecuencias a partir de las características sociodemográficas. Se estableció la prevalencia para la infección de la Enfermedad de Chagas, a través de la prueba de tamización y la complementaria. Para el análisis bivariado se comparó la prevalencia del marcador de infección, según sexo, tipo de donante y procedencia, utilizando chi cuadrado de Fisher. El procesamiento y análisis estadístico se realizó mediante el software $\mathrm{R}$ Project.

\section{Componente ético}

Según la resolución colombiana 8430 de 1993 del Ministerio de Salud el estudio se considera una investigación sin riesgo. La obtención de la información se ajustó a las normas éticas de garantía de la confidencialidad, de los beneficios y del riesgo mínimo para los participantes.

\section{Resultados}

Se evaluaron 25.920 donantes, 9187 durante el año 2016; 8517 en el 2017 y 8216 en el 2018, de los cuales $56,1 \%$ eran de sexo femenino y $43,9 \%$ masculino, el rango de edad en el que se presentaron más donantes fue de 18 a 30 años con 47,1\%. En la prueba de tamización para infección por $T$. cruzi la prevalencia fue de $0,17 \%(45 / 25.920)$ de ellos $77,0 \%$ eran mujeres y el $80,1 \%$ do-nantes voluntarios por primera vez. El rango de edad en el que se presentó mayor prevalencia fue de 18 a 30 años con un $33,3 \%$. El mayor número de donantes era procedente de la ciudad de Duitama con 22,2\% segui-do por donantes procedentes de otros departamentos con 17,7\%.(Tabla $1)$.

\begin{tabular}{|c|c|c|c|c|}
\hline \multicolumn{2}{|c|}{ Variable } & $\mathbf{n}$ & $\%$ & $95 \%$ IC \\
\hline \multirow{3}{*}{ Año } & 2016 & 18 & 40,0 & $(26,67-56,16)$ \\
\hline & 2017 & 5 & 11,1 & $(0-27,27)$ \\
\hline & 2018 & 22 & 48,8 & $(35,56-65,05)$ \\
\hline \multirow{3}{*}{ Sexo } & Femenino & 34 & 75,5 & $(64,44-87,98)$ \\
\hline & Masculino & 11 & 24,4 & $(13,3-36,8)$ \\
\hline & 18-30 años & 15 & 33,3 & $(20-49,6)$ \\
\hline \multirow{3}{*}{ Edad } & 31-40 años & 11 & 24,4 & $(11,1-40,7)$ \\
\hline & 41-50 años & 9 & 20,0 & $(6,6-36,3)$ \\
\hline & 51-65 años & 10 & 22,2 & $(8,8-38,5)$ \\
\hline \multirow{3}{*}{$\begin{array}{l}\text { Tipo de } \\
\text { donante }\end{array}$} & $\begin{array}{l}\text { Voluntario } \\
\text { primera vez }\end{array}$ & 36 & 80,0 & $(71,1-92,3)$ \\
\hline & $\begin{array}{l}\text { Voluntario no } \\
\text { repetitivo }\end{array}$ & 8 & 17,7 & $(8,8-30,1)$ \\
\hline & $\begin{array}{l}\text { Voluntario } \\
\text { repetitivo }\end{array}$ & 1 & 2,2 & $(0-14,5)$ \\
\hline \multirow{8}{*}{ Procedencia } & Tunja & 6 & 13,3 & $(2,2-28,0)$ \\
\hline & Duitama & 10 & 22,2 & $(11,1-36,9)$ \\
\hline & Paipa & 2 & 4,4 & $(0-19,1)$ \\
\hline & Sogamoso & 6 & 13,3 & $(2,2-28,0)$ \\
\hline & Chiquinquirá & 3 & 6,7 & $(0-21,3)$ \\
\hline & Moniquirá & 3 & 6,7 & $(0-21,3)$ \\
\hline & $\begin{array}{c}\text { Otros } \\
\text { municipios de } \\
\text { Boyacá }\end{array}$ & 7 & 15,6 & $(4,4-30,7)$ \\
\hline & $\begin{array}{c}\text { Otros } \\
\text { Departamentos }\end{array}$ & 8 & 17,8 & $(6,6-32,4)$ \\
\hline
\end{tabular}

Tabla 1. Características epidemiológicas de donantes de sangre reactivos para anticuerpos anti- T. cruzi, Boyacá, Colombia $2016-2018(\mathrm{n}=45)$

La prevalencia con la prueba complementaria fue de $0,08 \%(20 / 25.920)$ del cual $70,0 \%$ eran mujeres, el $85,0 \%$ donantes voluntarios por primera vez, el rango de edad en el que se presentó mayor prevalencia de este marcador serológico fue de 41 a 50 y de 51 a 65 años con un 35,0\% cada uno (Tabla 2). En el año 2017 no se presentaron casos positivos. 


\begin{tabular}{|c|c|c|c|c|}
\hline \multicolumn{2}{|c|}{ Variable } & $\mathbf{n}$ & $\%$ & $95 \%$ IC \\
\hline \multirow{2}{*}{ Año } & 2016 & 7 & 35 & $(20-58.58)$ \\
\hline & 2018 & 13 & 65 & $(50-88.58)$ \\
\hline \multirow{2}{*}{ Sexo } & Femenino & 14 & 70 & $(55-91.86)$ \\
\hline & Masculino & 6 & 30 & $(15-51.86)$ \\
\hline \multirow{4}{*}{ Edad } & 18-30 años & 2 & 10 & $(0-33.13)$ \\
\hline & 31-40 años & 4 & 20 & $(0-43.13)$ \\
\hline & 41-50 años & 7 & 35 & $(15-58.13)$ \\
\hline & 51-65 años & 7 & 35 & $(15-58.13)$ \\
\hline \multirow{3}{*}{$\begin{array}{l}\text { Tipo de } \\
\text { donante }\end{array}$} & $\begin{array}{l}\text { Voluntario } \\
\text { primera vez }\end{array}$ & 17 & 85 & $(75-100.00)$ \\
\hline & $\begin{array}{l}\text { Voluntario no } \\
\text { repetitivo }\end{array}$ & 2 & 10 & $(0-26.07)$ \\
\hline & $\begin{array}{l}\text { Voluntario } \\
\text { repetitivo }\end{array}$ & 1 & 5 & $(0-21.07)$ \\
\hline \multirow{7}{*}{ Procedencia } & Tunja & 3 & 15 & $(0-39.91)$ \\
\hline & Moniquirá & 3 & 15 & $(0-39.91)$ \\
\hline & Sogamoso & 1 & 5 & $(0-29.91)$ \\
\hline & Chiquinquirá & 1 & 5 & $(0-29.91)$ \\
\hline & Duitama & 1 & 5 & $(0-29.91)$ \\
\hline & $\begin{array}{c}\text { Otros municipios } \\
\text { de Boyacá }\end{array}$ & 4 & 20 & $(5-44.91)$ \\
\hline & $\begin{array}{c}\text { Otros } \\
\text { Departamentos }\end{array}$ & 7 & 35 & $(20-59.91)$ \\
\hline
\end{tabular}

Tabla 2. Características epidemiológicas de donantes de sangre confirmados para Chagas. Boyacá, Colombia $2016-2018(n=20)$

Fuente: autores
En la tabla 3, se evidencia que la prevalencia de la infección de Chagas presentó asociación estadísticamente significativa con la edad $(p<0,05)$ con mayor presencia en los grupos de 41-50 y 51-65 años.

\begin{tabular}{|c|c|c|c|c|c|c|c|}
\hline \multirow[t]{3}{*}{ Variable } & \multicolumn{5}{|c|}{ Resultado } & \multirow{3}{*}{ Chi 2} & \multirow{3}{*}{$p$} \\
\hline & \multirow{2}{*}{$\begin{array}{l}\text { Positivo } \\
\#\end{array}$} & \multicolumn{3}{|c|}{ Negativo } & & & \\
\hline & & $\%$ & $\#$ & $\%$ & & & \\
\hline \multirow{2}{*}{ Sexo } & Mujeres & 14 & 70 & 20 & 80 & \multirow[b]{2}{*}{0,4548} & \multirow{2}{*}{0,5001} \\
\hline & Hombres & 6 & 30 & 5 & 20 & & \\
\hline \multirow{4}{*}{ Edad } & 18-30 años & 2 & 10 & 13 & 52 & \multirow{4}{*}{13,0436} & \multirow{4}{*}{0,0045} \\
\hline & 31-40 años & 4 & 20 & 7 & 28 & & \\
\hline & 41-50 años & 7 & 35 & 2 & 8 & & \\
\hline & 51-65 años & 7 & 35 & 3 & 12 & & \\
\hline \multirow{3}{*}{$\begin{array}{l}\text { Tipo de } \\
\text { donante }\end{array}$} & $\begin{array}{l}\text { Voluntario } \\
\text { primera vez }\end{array}$ & 17 & 85 & 19 & 76 & \multirow{3}{*}{2,7239} & \multirow{3}{*}{0,2561} \\
\hline & $\begin{array}{l}\text { Voluntario } \\
\text { repetitivo }\end{array}$ & 2 & 10 & 6 & 24 & & \\
\hline & $\begin{array}{l}\text { Voluntario no } \\
\text { repetitivo }\end{array}$ & 1 & 5 & 0 & 0 & & \\
\hline
\end{tabular}

*Valor significativo $<0.05$

Tabla 3. Asociación de la prevalencia de infección por T. cruzi con la prueba complementarias en donantes de sangre de Boyacá, según las características epidemiológicas

En cuanto a los donantes confirmados, el 55,0\% eran del departamento de Boyacá y el $45 \%$ de otros departamentos como Casanare, Meta, Santander, Norte de Santander y Córdoba (Figura 1) 


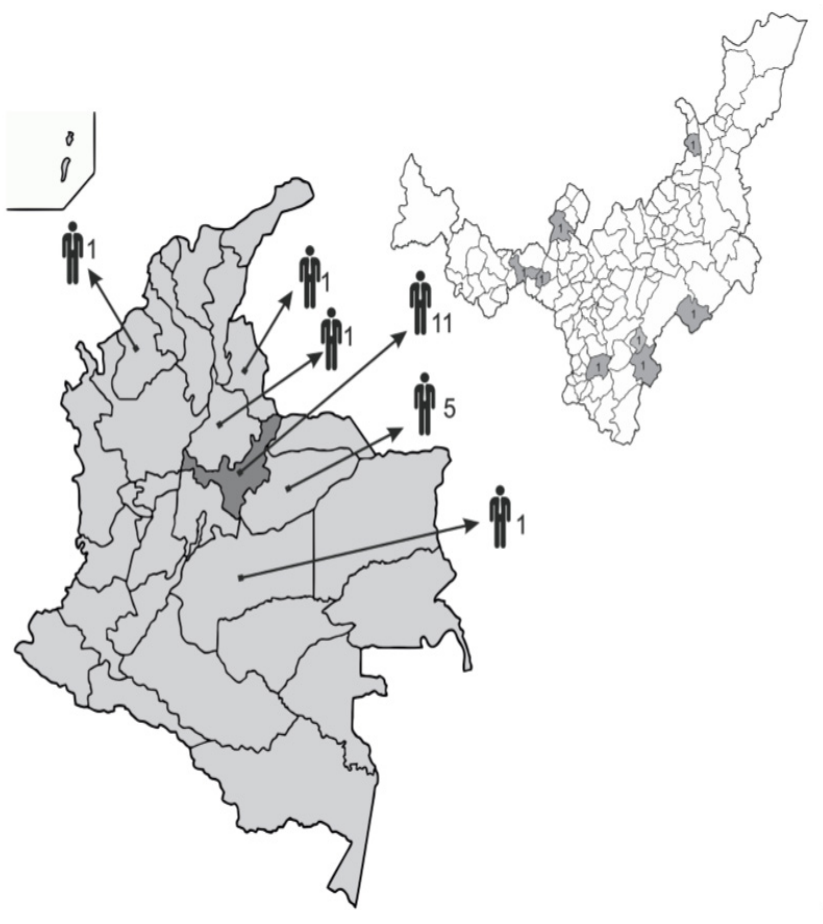

Figura 1. Mapa de Colombia de distribución geográfica de casos positivos confirmados para anti-T. anticuerpos cruzi

\section{Discusión}

Los resultados del presente estudio mostraron una prevalencia de anticuerpos contra Trypanosoma cruzi en el tamizaje de $0.17 \%$ y en la prueba complementaria fue de $0,08 \%$, similar a la hallada en Barranquilla que fue de $0.27 \%$ y $0.03 \%$ (14) y en Córdoba de $0.43 \%$ y $0.39 \%$ (15) respectivamente, pero inferior a lo reportado a nivel nacional $(10,11,12)$.

El departamento de Boyacá cuenta con una certificación internacional para la eliminación de la transmisión en el $39.3 \%$ de los municipios, lo que a permitido disminuir la incidencia de la EC en zonas de riesgo.

Los datos obtenidos del comportamiento de este evento en el Banco de sangre del departamento de Boyacá, pueden explicarse debido al estricto cumplimiento de los lineamientos del nivel nacional referentes a la donación voluntaria de sangre, en los cuales se busca identificar factores de riesgo que puedan vulnerar la salud del donante y del receptor (16) para que, al momento de la donación de sangre, se realice una selección adecuada y efectiva. Además, han impactado de manera positiva las acciones de prevención y control vectorial realizadas por la Secretaría de Salud de Boyacá, fortalecidas con intervenciones químicas e integrales que involucran la participación comunitaria, haciendo que la población sea más consciente e identifique su exposición al riesgo.

En cuanto a la variable de género, se encontró que la población femenina fue la que realizó mayor donación con un valor porcentual del $56.1 \%$. Cabe señalar al respecto, que la OMS recomienda que las mujeres no realicen de manera recurrente donación de sangre, con el fin de evitar una posible deficiencia de hierro en su organismo (16). En cuanto a la prueba complementaria, el sexo femenino presentó mayor frecuencia con $70.0 \%$, diferente a lo descrito en Caquetá que fue $74.0 \%$ de hombres (17) y en otras regiones del mundo como en Brasilia con $63.4 \%(18)$.

El rango de edad con mayor frecuencia de donantes positivos para Chagas en el presente estudio estuvo entre 41-51 años y 51-65, diferente a lo reportado en estudios realizados en Venezuela (19) y Perú (20) en los que el rango de edad que mayor prevalencia presentó fue de 18-31 años con 50.4\% y 33.3\%, respectivamente. En el estudio realizado en Boyacá, se encontró asociación estadísticamente significativa entre la edad y la seropositividad similar a lo reportado en un estudio realizado en México (21) y coincidiendo con lo observado en estudios de población abierta, donde la seroprevalencia aumenta considerablemente en la cuarta y quinta década de vida (22).

En cuanto al tipo de donantes, los donantes voluntarios por primera vez presentaron un mayor porcentaje de positividad para la infección por T. cruzi con $85 \%$, similar a lo descrito en un estudio realizado en Brasil en el Servicio de Hemoterapia en el que fue del 75.3\% (23) y en el Centro de Uberaba Fundación Hemominas que fue de $99.2 \%$ y en el que encontraron asociación significativa $(\mathrm{OR}=607 ; 84.9-4348.3)$ (24), diferente a lo descrito en el presente estudio en el cual no se encontró asociación significativa. Los donantes voluntarios repetitivos son previamente seleccionados ya que han sido sometidos a cribadio clínico y serológico en donaciones anteriores, y por tanto, tienen tasas de seropositividad más bajas (25), coincidiendo con lo encontrado en el presente estudio.

Los bancos de sangre resultan ser una de las fuentes de información disponibles para monitorear el comportamiento de la enfermedad de Chagas y evaluar la toma de desiciones en salud pública ya que la mayoría de los casos son asintomáticos y sin compromiso clínico. La normatividad que rige a los bancos de sangre frente a la tamización y pruebas complementarias para marcadores serológicos, entre ellos anti $T$ cruzi, facilita el fortalecimiento de la ruta de atención integral en salud implementada en el departamento, la búsqueda activa y la canalización efectiva a través de los actores 
del sistema de salud, permitiendo realizar diagnóstico complementario, seguimiento y tratamiento, dando cumplimiento a los altgoritmos y normatividad vigente relacionados.

Por último, cabe señalar que en el departamento de Boyacá las acciones de prevención y control de las enfermedades transmitidas por vectores, especialmente la enfermedad de Chagas, han sensibilizado a la población frente a los factores determinantes de la presencia de la enfermedad, lo que le permite al banco de sangre contar con un proceso de selección de donantes más confiable y efectivo.

\section{Agradecimientos}

A los profesionales y personal de apoyo en salud del Hemocentro del Centro Oriente Colombiano.

\section{Conflicto de interés}

Los autores no manifiestan conflictos de intereses.

\section{Referencias}

1. Instituto Nacional de Salud. Guía Protocolo para la vigilancia en salud pública de Chagas. [Internet] 2017 [citado el 10 de diciembre 2020]. Disponible en: https://www.minsalud. gov.co/Documents/Salud\%20P\%C3\%BAblica/ Ola\% 20 invernal/Protocolo\%20 Chagas.pdf

2. Cucunubá Z. Mecanismos de transmisión de la enfermedad de Chagas. 2009. Ministerio de la protección Social.

3. Storino R, Auger S, Caravello O, Urrutia MI, Sanmartino M, Jörg M. Cardiopatía chagásica en pacientes de área endémica versus contagiados en forma ocasional. Rev Saúde Pública. [Internet] 2002[citado el 10 de diciembre 2020]; 36:755-8. Disponible en: https://doi.org//10.1590/ S0034-89102002000700016

4. Requena-Méndez A, Aldasoro E, de Lazzari E, Sicuri E, Brown M, Moore DA, Gascon J, Muñoz J. Prevalence of Chagas disease in LatinAmerican migrants living in Europe: a systematic review and meta-analysis. PLoS Negl Trop Dis. 2015;9(2):e0003540.doi: 10.1371/journal.pntd.0003540.

5. Barona-Vilar C, Giménez-Martí MJ, Fraile T, González-Steinbauer C, Parada C, Gil-Brusola A, Bravo D, Gómez MD, Navarro D, Perez-Tamarit A, FernandezSilveira L, Fullana-Montoro A, Borrás R. Prevalence of Trypanosoma cruzi infection in pregnant Latin American women and congenital transmission rate in a non-endemic area: the experience of the Valencian Health Programme (Spain). Epidemiol Infect. 2012 Oct;140(10):1896903. doi: $10.1017 /$ S0950268811002482.

6. Pinazo MJ, Gascon J. The importance of the multidisciplinary approach to deal with the new epidemiological scenario of Chagas disease (global health). Acta Trop. 2015 Nov;151:16-20. doi: 10.1016/j.actatropica.2015.06.013.

7. Organización Mundial de la Salud. La enfermedad de Chagas, tripanosomiais americana. [Internet] 2019 [citado el 10 de diciembre 2020]. Disponible en: http://www.who.int/es/news-room/fact-sheets/ detail/chagas-disease-(american-trypanosomiasis)

8. Instituto Nacional de Salud. Informe del evento enfermedad de Chagas, Colombia, año 2016. [Internet] 2016 [citado el 10 de diciembre 2020]. Disponible en: https://www.ins.gov.co/buscadoreventos/Informesdeevento/Chagas\%202016.pdf

9. Ministerio de Salud y Proyección Social. Instituto Nacional de Salud. Informe del evento Chagas, Colombia, año 2017. [Internet] 2017 [citado el 10 de diciembre 2020]. Disponible en: https://www.ins.gov.co/buscadoreventos/Informesdeevento/CHAGAS\%20\%202017.pdf

10. Instituto Nacional de Salud. Informe anual red nacional de bancos de sangre y servicios de transfusión, Colombia 2016. [Internet] 2016 [citado el 10 de diciembre 2020]Disponible en: https://www.ins.gov.co/buscadoreventos/Informesdeevento/CHAGAS\%20\%202017.pdf

11. Instituto Nacional de Salud. Informe anual red nacional de bancos de sangre y servicios de transfusión, Colombia 2017. [Internet] 2017 [citado el 10 de diciembre 2020] Disponible en: https://www.ins.gov.co/Direcciones/RedesSaludPublica/DonacionSangre/AreasEstrategicas/Informe\%20anual\%20Red\%20Sangre\%20 2017\%20v2.pdf

12. Instituto Nacional de salud. Informe ejecutivo de la red nacional bancos de sangre, Colombia 2018. [Internet] 2018 [citado el 10 de diciembre 2020] Disponible en: https://www.ins.gov.co/Direcciones/RedesSaludPublica/DonacionSangre/AreasEstrategicas/ informe-ejecutivo-bancos-de-sangre-colombia-2018.pdf

13. Instituto Nacional de Salud. Circular 0081 del 9 de agosto de 2011. Donación voluntaria de sangre. [Internet] 2011 [citado el 10 de diciembre 2020] Disponible en: https://www.ins.gov.co/BibliotecaDigital/Circular- 
081-de-2011.pdf

14. Camargo De la Hoz L, Consuegra C, Coronado A, Tenorio E, Becerra J, Sarmiento-Rubiano LA. Perfil de los donantes de un Banco de sangre de la ciudad de Barranquilla-Colombia, años 2014 y 2015. Archivos Venezolanos de Farmacología y Terapéutica. 2018;37(3):227-234

15. Ruiz -Mendoza L, Villegas- Gracia R, CardonaArias J. Prevalencia de agentes transmisibles por transfusión y factores asociados en un banco de sangre de Córdoba- Colombia 2014-2016. Rev.udcaactualdivulg. cient. [Internet]. 2018 Dec [citado el 10 de diciembre 2020]; 21( 2 ): 297-308. Disponible en: http://www. scielo.org.co/scielo.php?script=sci_arttext\&pid=S0123$42262018000200297 \& \operatorname{lng}=$ en .

https:// doi.org/10.31910/rudca.v21.n2.2018.969.

16. Ministerio de Salud y Proyección Social. Instituto Nacional de Salud. Lineamiento técnico para la selección de donantes de sangre en Colombia- Documento Técnico.[Internet] 2018 [citado el 10 de diciembre 2020].Disponible en: https://www.ins. gov.co/Direcciones/RedesSaludPublica/DonacionSangre/Publicaciones/Lineamiento\%20tecnico\%20 Selecci\%C3\%B3n\%20de\%20donantes\%202018.pdf

17. Mauricio Beltrán-Durán, Liliana B. Hilarión-Gaitán, Maritza Berrío-Pérez y María I. Bermúdez. Detección de anticuerpos para Trypanosoma cruzi en donantes de sangre. Caquetá, Colombia, 1995 a 2010. Rev. Salud Pública. 2017; 19(3):355-361

18. SantanaMP,Souza-SantosR,AlmeidaAS.Factors associated with Chagas disease among blood donorsin Brazilian Northeast region. J Infect Public Health. 2018 NovDec;11(6):817-820. doi: 10.1016/j.jiph.2018.06.001.

19. Barrueta MC, González CA, Bolívar AM. Trypanosoma cruzi en donantes que acuden al banco de sangre "Dr. Julio García Álvarez" del hospital Dr. Luis Razetti, estado Barinas, Venezuela. Kasmera. 2019;47(2):102-107. doi: 10.5281/zenodo.3522044

20. Charres-Coaguila P, Novoa-Ávalos P. Seroprevalencia y características epidemiológicas de la enfermedad de chagas en donantes de sangre que acudieron al hospital Hipólito Unanue de Tacna 2013-2017 [Tesis Doctoral]. Perú: Facultad de Medicina. Univ Privada de Tacna; 2019. Disponible en: http://repositorio.upt.edu. pe/bitstream/UPT/660/1/Charres-Coaguila-Patricio.pdf

21. Monteón VM, Reyes-López PA, SosaPalacio A, León-Tello G, Martínez-Murguía,
Sosa-Jurado F. Distribución heterogénea de la prevalencia de anticuerpos contra Trypanosoma cruzi en donadores de sangre en Puebla, México. Salud pública Mex. 2005; 47(2)

22. Sosa-Jurado F, Zumaquero-Ríos JL, Reyes PA, Cruz-García A, Guzmán-Bracho C, Monteón VM. Factores bióticos y abióticos que determinan la seroprevalencia de anticuerpos contra Trypanosoma cruzi en el municipio de Palmar de Bravo, Puebla, México. Salud Pública Mex. 2004; 46: 39-48.

23. Santana MP, et al. Factors associated with Chagas disease among blood donors in Brazilian Northeast region. J Infect Public Health. 2018. $\quad$ https://doi.org/10.1016/j.jiph.2018.06.001

24. Ferreira-Silva MM, Pereira GA, Lages-Silva E, Moraes-Souza M. Cribado socioepidemiológico de donantes de sangre serológicamente no elegibles por enfermedad de Chagas para la definición de casos no concluyentes. Mem Inst Oswaldo Cruz. 2010; 105(6): 800-805

25. Wendel S. Doença de chagas transfusional. En: Dias JCP y Coura JR, org. Clínica e terapêutica da doença de Chagas: uma abordagem prática para o clínico geral [Internet]. Rio de Janeiro: Editora FIOCRUZ;1997[citado el 10 de diciembre 2020]. Disponible en: http://books.scielo.org/id/nf9bn/pdf/dias9788575412435-24.pdf 